Article

\title{
Entanglement, Complementarity, and Vacuum Fields in Spontaneous Parametric Down-Conversion
}

\author{
Ralf Menzel ${ }^{1}$, Axel Heuer ${ }^{1}$ and Peter W. Milonni ${ }^{2,3, *}$ \\ 1 Institute of Physics and Astronomy, University of Potsdam, Karl-Liebknecht Straße 24-25, D-14476 Potsdam, \\ Germany; menzel@uni-potsdam.de (R.M.); heuer@uni-potsdam.de (A.H.) \\ 2 Theoretical Division, Los Alamos National Laboratory, Los Alamos, NM 87545, USA \\ 3 Department of Physics and Astronomy, University of Rochester, Rochester, NY 14627, USA \\ * Correspondence: peter_milonni@comcast.net
}

Received: 16 January 2019; Accepted: 12 February 2019; Published: 19 February 2019

check for updates

\begin{abstract}
Using two crystals for spontaneous parametric down-conversion in a parallel setup, we observe two-photon interference with high visibility. The high visibility is consistent with complementarity and the absence of which-path information. The observations are explained as the effects of entanglement or equivalently in terms of interfering probability amplitudes and also by the calculation of a second-order field correlation function in the Heisenberg picture. The latter approach brings out explicitly the role of the vacuum fields in the down-conversion at the crystals and in the photon coincidence counting. For comparison, we show that the Hong-Ou-Mandel dip can be explained by the same approach in which the role of the vacuum signal and idler fields, as opposed to entanglement involving vacuum states, is emphasized. We discuss the fundamental limitations of a theory in which these vacuum fields are treated as classical, stochastic fields.
\end{abstract}

Keywords: complementarity; vacuum fields; entanglement; Hong-Ou-Mandel effect; spontaneous parametric down-conversion

PACS: $42.50 . \mathrm{Ar} ;$ 42.50.Dv

\section{Introduction}

Entanglement is of interest not only as a basic feature of quantum theory, but also for applications in quantum optics including quantum cryptography [1], quantum imaging [2], quantum spectroscopy [3,4], and metrology [5], among others. The strong correlations implied by entanglement have long been of interest in connection with quantum interference effects [6] and various classically counterintuitive experimental results, and there have been many investigations of entanglement with suitable experiments and associated theoretical analyses.

Entanglement contains some of the surprising features of quantum theory and demonstrates correlations stronger than are possible in classical physics. Different approaches were taken in the past, such as the introduction of hidden parameters, to explain these observations. However, it was shown that such hidden parameters are inconsistent with quantum theory and experiment. As we show in this paper, the explicit consideration of vacuum quantum fields allows an intuitive description of quantum-optical effects in spontaneous parametric down-conversion that are typically explained by entanglement.

Spontaneous parametric down-conversion (SPDC) has been one of the most useful tools in quantum-optical investigations of entanglement. In this paper we revisit SPDC from a perspective somewhat different from much of the previous work on the subject. For this purpose, we investigated the interference of two biphotons generated in two parallel, pumped down-conversion crystals as 
in [7], but with no further coupling, as in [8,9]. Our experimental results are in agreement with the earlier work (but with much greater interference visibility), but it seems worthwhile to describe and analyze them from the perspectives taken in our recently-reported work $[7,10]$ and to formulate in more detail the analyses outlined in that work. In doing so, the question arises as to the extent to which the experimental results we show can be understood in a classical, stochastic treatment of the vacuum field. We discuss the difficulties posed by such an intuitive approach to the signal and idler vacuum fields, which can nevertheless account for some of the effects observed in SPDC [11].

In analyzing our earlier experiments, we considered in particular the role of vacuum fields and complementarity in different SPDC setups. SPDC is especially interesting in this regard because the down-converted photons result from nonlinear mixing of the pump field with the vacuum fields at the crystals, and therefore, the statistical properties of the generated signal and idler fields reflect those of the vacuum fields involved in the three-wave mixing process. It has been demonstrated that, in spite of the random character of these vacuum fields, a phase memory effect could be observed if the same vacuum mode takes part in the down-conversion in two crystals $[9,12]$. Using an extended version of this experiment with three down-conversion crystals, it was shown that the absence of correlations between the vacuum fields at different crystals results in an incoherent background photon count. However, this effect of the different vacuum field modes taking part in the down-conversion could be completely overcome, and almost perfect coherence could be observed, in the stimulated process with coherent laser radiation, distinct from the pump laser, fed into a previously open vacuum mode [7].

We begin in the following section by describing the experiment with two down-conversion crystals. In this experiment, there are a total of four open vacuum entrances, two at each crystal, involved in the three-wave-mixing processes generating the two down-converted biphotons. No first-order coherence is observed between the single-photon signal and idler fields, but high-visibility interference is observed when photons are counted in coincidence, consistent with complementarity and the absence of which-path information (Section 3). In Section 4, a Heisenberg picture analysis of these results is presented. In this description, there is no first-order coherence observed between the single-photon signal and idler fields because the vacuum fields at the four open entrances are uncorrelated, whereas second-order, "intensity" correlations of these vacuum fields result in the observed interference effect when photons are counted in coincidence. The same formalism is then used in Section 5 to describe the Hong-Ou-Mandel (HOM) dip [13], where we show that this consequence of entanglement and quantum interference can again be explained equivalently in terms of non-vanishing intensity correlations of the vacuum fields. The vacuum signal and idler fields in both the two-crystal experiment and the HOM effect make explicit contributions not only to the generation of the signal and idler photons, but also to the photon coincidence counting rate. In Section 6, we discuss further the simplified Heisenberg picture theory employed in Sections 4 and 5 and its relation to a more complete SPDC theory. Section 7 addresses the question of the extent to which the vacuum field in these experiments can be regarded as a classical stochastic field. Our results are summarized and discussed further in Section 8.

\section{Experiment}

The experimental setup consisted of two Beta Barium Borate crystals (BBO1 and BBO2) for the generation of the down-converted signal and idler photon pairs. The crystals had a length of $4 \mathrm{~mm}$ and were arranged in parallel. As shown in Figure 1, the signal channel s1 of BBO1 is overlaid with the signal channel s2 of $\mathrm{BBO} 2$ at the beam splitter BS1, and the idler channel i1 is overlaid with the idler channel i2 at the beam splitter BS2.

The single signal photons were counted at the peak wavelength of $808 \mathrm{~nm}$ using a filter with a bandwidth (FWHM) of $2 \mathrm{~nm}$, and the idler photons were counted at the peak wavelength of $632 \mathrm{~nm}$ with a bandwidth of $3 \mathrm{~nm}$. The two cw, 355-nm nearly diffraction-limited pump fields from the laser (Genesis, Coherent, Inc., Santa Clara, CA, USA) had a bandwidth of about $45 \mathrm{GHz}$, corresponding to a coherence length of about $1.5 \mathrm{~mm}$, and were synchronized, but could be delayed with respect 
to each other with a delay line in front of BBO1. To vary the length of the signal and the idler single-photon interferometers, the $100 \%$ reflecting mirrors could be moved, resulting in phase shifts for the signal fields (upper mirror in Figure 1) and for the idler fields (lower mirror in Figure 1). Photons were counted with single-mode, fiber-coupled photodiodes (SPCM-AQRH-13, Perkin Elmer, Inc., Waltham, MA, USA) at Positions A and B. The whole setup was aligned with an Electron Multiplying Charge Coupled Device (EMCCD) camera (Ixon, Andor, Inc., Belfast, UK) at Positions A and B. The pump power at each crystal was about $30 \mathrm{~mW}$. Photon count rates at Detectors A and B were 25,000 photons/s from crystal BBO1 and 26,000 photons/s from BBO2. With a high probability, no more than one photon pair was in the apparatus during the measurement interval of $2 \mathrm{~ns}$, so that stimulated down-conversion could be neglected in these experiments. All the observed interference effects were based on the coincidence counting of a single photon pair, either from $\mathrm{BBO} 1$ or from $\mathrm{BBO} 2$.

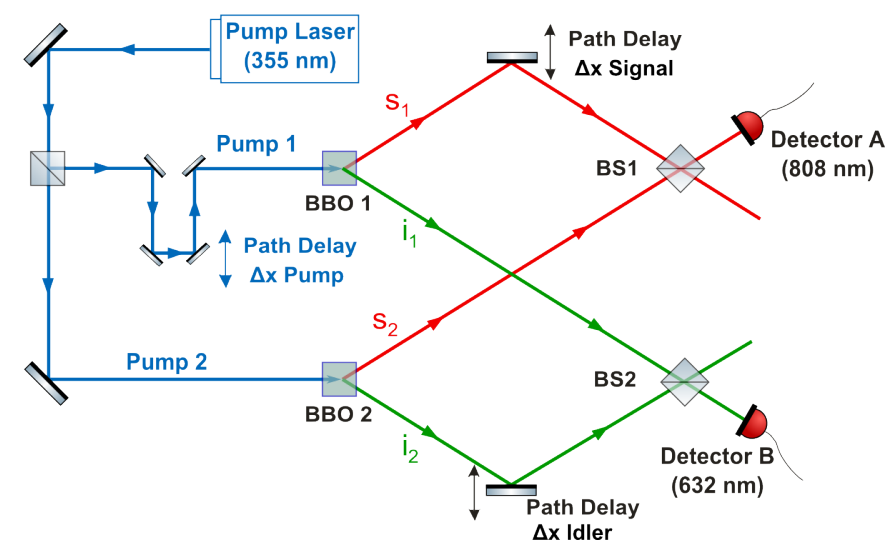

Figure 1. Two-photon interference setup with two synchronously-pumped spontaneous parametric down-conversion (SPDC) crystals BBO1 and BBO2. The rate of counting signal and idler photons in coincidence at Detectors A and B is measured as a function of the path delay of the pump field. The pump intensity is sufficiently weak such that with high probability, only one signal-idler photon pair is present during a measuring period. BS, beam splitter.

\section{Results}

In the first experiment, the coincidence count rate was measured as a function of the signal path delay. The result is shown in Figure 2.

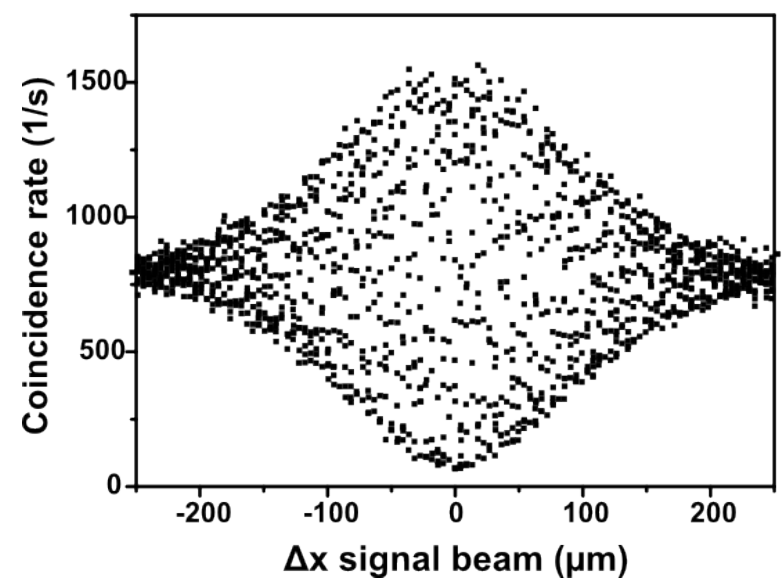

Figure 2. Counting rate for single signal photons at Detector $\mathrm{A}$ in coincidence with single idler photons at Detector B as a function of the signal path delay (see Figure 1).

This measurement was done over a wide range of delays in order to determine the coherence length of the fields. From Figure 2, the coherence length of the signal field can be estimated to be 
$80 \mu \mathrm{m}$, in good agreement with the bandwidth of the spectral filter; the coherence length of one of the biphoton fields determines the coherence length in the second-order interference measurement. In a second measurement, the spatial resolution of the delay line was increased in order to exhibit the biphoton interference in more detail, as shown in Figure 3.

The "fringe" spacing in this biphoton interference measurement is in very good agreement with the 808-nm wavelength of the signal field. From the data shown in Figure 3, the maximum visibility at zero delay was $94 \%$. Similar results were obtained when the path delay for the idler photons was varied. In that case, the fringe spacing in the biphoton interference is in agreement with the idler photon wavelength of $632 \mathrm{~nm}$. Since both crystals were "coupled" only by the pump laser radiation, the observed biphoton interference with visibility $\cong 1$ may seem surprising.

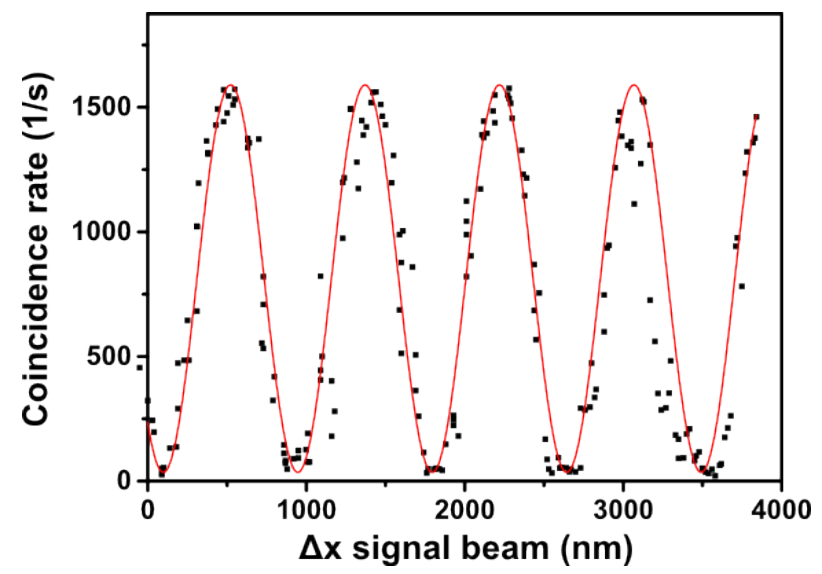

Figure 3. Counting rate for single signal photons at Detector A in coincidence with single idler photons at Detector B as a function of the signal path delay as in Figure 2, but with higher resolution.

The influence of the pump is shown by also varying Pump 1 path delay (Figure 4 ). It is seen that the visibility of the biphoton interference is now even higher, with a value of $\mathrm{V}=98 \%$. The fringe spacing is the wavelength of the pump laser, confirming the phase memory effect [9,12] in this measurement. The coherence length evaluated from this type of measurement, over a wide range of path delays, is the coherence length of the pump laser, $1.5 \mathrm{~mm}$.

In summary, the second-order interference measurement of the biphotons emitted from two parallel, pumped, and otherwise uncoupled down-conversion crystals BBO1 and BBO2 shows a fringe visibility $\cong 1$. The fringe spacing observed in these measurements always corresponds to the wavelength of the delayed field.

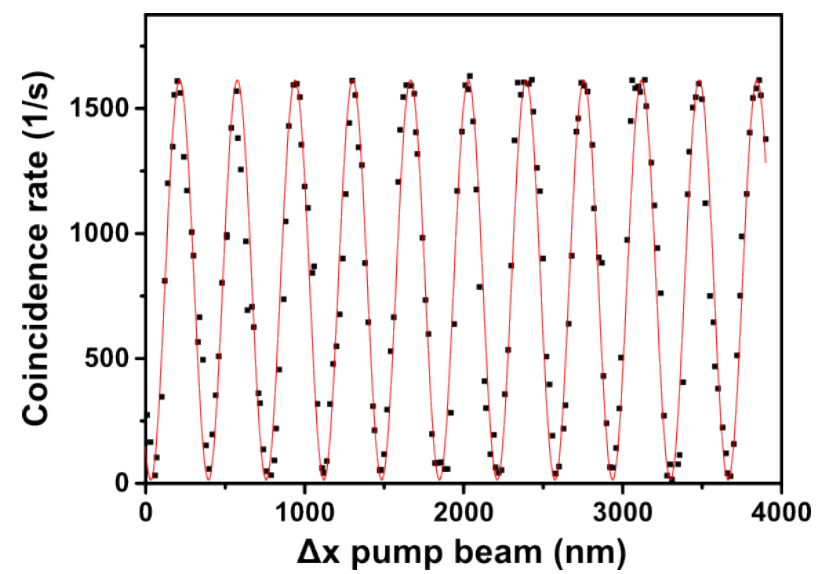

Figure 4. Second-order interference of the single signal photons from crystals $\mathrm{BBO} 1$ or $\mathrm{BBO} 2$ at Detector A in coincidence with the single idler photons from crystals BBO1 or BBO2 at Detector B, as a function of Pump 1 path delay for BBO1 (see Figure 1). 


\section{Theoretical Description}

The interaction Hamiltonian describing the down-conversion process in a single crystal is

$$
H_{I}=C a_{s}^{\dagger} a_{i}^{\dagger} e^{-i \omega_{p} t}+C^{*} a_{s} a_{i} e^{i \omega_{p} t}
$$

when the pump is treated as a classically-prescribed, undepleted, and spatially-uniform field of frequency $\omega_{p} . a_{s}$ and $a_{i}$ are the photon annihilation operators for the signal and idler fields satisfying the energy conservation $\left(\omega_{p}=\omega_{s}+\omega_{i}\right)$ and phase-matching $\left(\mathbf{k}_{p}=\mathbf{k}_{s}+\mathbf{k}_{i}\right)$ conditions, and $C$ is proportional to the pump amplitude and the nonlinear susceptibility. To lowest order in $C$, $H_{I}$ in spontaneous parametric down-conversion generates an entangled field state $[9,14]$ that is a linear superposition of the vacuum state $\left|0_{s} 0_{i}\right\rangle$ and the two-photon state $\left|1_{s} 1_{i}\right\rangle$ consisting of a single-signal photon and a single-idler photon. In the Heisenberg picture, the positive frequency (photon annihilation) parts of the signal and idler electric field operators may be expressed, to lowest order in $C$, as

$$
E_{s}^{(+)}(\mathbf{r}, t)=a_{s}(t) U_{s}(\mathbf{r}) \cong\left[a_{s 0}+D a_{i 0}^{\dagger}\right] U_{s}(\mathbf{r}) e^{-i \omega_{s} t},
$$

and

$$
E_{i}^{(+)}(\mathbf{r}, t)=a_{i}(t) U_{i}(\mathbf{r}) \cong\left[a_{i 0}+D a_{s 0}^{\dagger}\right] U_{i}(\mathbf{r}) e^{-i \omega_{i} t} .
$$

$a_{s 0}$ and $a_{i 0}$ are the photon annihilation operators for the signal and idler vacuum fields, respectively, in the absence of the pump field. The functions $U_{\mathcal{S}}(\mathbf{r})$ and $U_{i}(\mathbf{r})$ include the spatial dependence of the corresponding electric fields, which are polarized according to type I phase matching in the experiments described in the preceding sections. $D$, which is proportional to $C$, is determined by the solution of the Heisenberg equations of motion that follow from the Hamiltonian with the interaction (1). The specific forms of $U_{s}(\mathbf{r}), U_{i}(\mathbf{r})$, and $D$ will not be needed for our purposes.

As illustrated in Figure 5, the signal field $E_{s}^{(+)}$in our simplified Heisenberg picture analysis has two contributions: (i) the vacuum field in the signal mode and (ii) the field generated by the mixing in the crystal of the pump field with the vacuum field in the idler mode. Likewise, the idler field $E_{i}^{(+)}$ consists of the vacuum field in the idler mode plus the field generated by the mixing in the crystal of the pump field with the vacuum field in the signal mode.

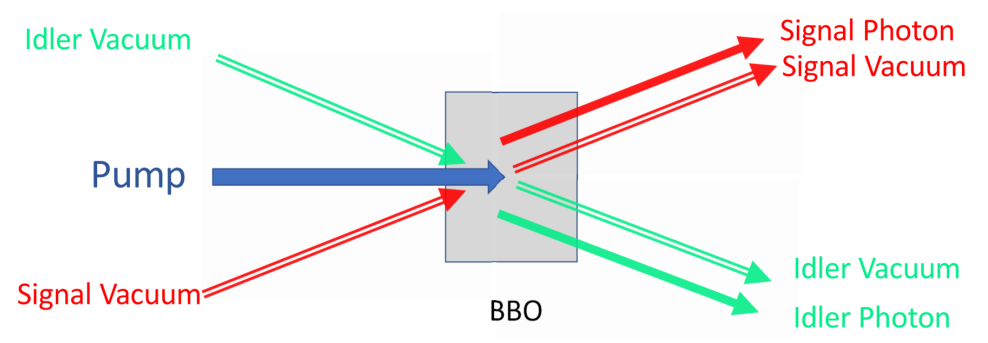

Figure 5. Signal- (idler-) photons are created by the mixing in the BBO crystal of the pump field with the vacuum field in the idler (signal) mode, as described by Equations (2) and (3). The signal (idler) field emerging from the crystal is the sum of the vacuum field in the signal (idler) mode and the signal (idler) field resulting from the nonlinear mixing process described by the Hamiltonian (1).

For the two-crystal configuration of interest here,

$$
H_{I}=\left[C_{1} a_{s 1}^{\dagger} a_{i 1}^{\dagger}+C_{2} a_{s 2}^{\dagger} a_{i 2}^{\dagger}\right] e^{-i \omega_{p} t}+\text { Hermitian conjugate, }
$$

where the subscripts s 1, s2, i1, and i2 refer to the modes indicated in Figure 1. The two crystals are assumed to be identical, in which case $C_{1}$ and $C_{2}$ are proportional to the complex amplitudes of the pump fields at $\mathrm{BBO} 1$ and $\mathrm{BBO} 2$, respectively. 
The coincidence rate may be obtained simply from the superposition of probability amplitudes. Suppose first that $C_{1}=C_{2} \equiv C$. There are two (indistinguishable) ways by which a signal photon can be counted at $A$ in coincidence with an idler photon at $B$. Referring to Figure 1, these are (i) a signal photon $\mathrm{s} 1$ is counted at $\mathrm{A}$ and an idler photon $\mathrm{i} 1$ at $\mathrm{B}$, and (ii) a signal photon $\mathrm{s} 2$ is counted at $\mathrm{A}$ and an idler photon $\mathrm{i} 2$ at B. In Process (i), the signal field undergoes a reflection and a phase delay $\phi_{1}$, and the idler field is transmitted through the lower beam splitter in Figure 1. The amplitude for Path (i) is therefore proportional to $r t e^{i \phi_{1}}$, where $r$ and $t$ are respectively the amplitude reflection and transmission coefficients of the beam splitters, which are assumed to be identical. In Process (ii), the signal field is transmitted through the upper beam splitter in Figure 1, the idler field undergoes a reflection and a phase delay $\phi_{2}$, and the probability for this path is therefore proportional to $t r e^{i \phi_{2}}$. The total probability amplitude for a signal photon to be counted at $\mathrm{A}$ in coincidence with an idler photon at $\mathrm{B}$ is therefore proportional to $r t\left[C e^{i \phi_{1}}+C e^{i \phi_{2}}\right]$, and the coincidence counting rate is proportional to $|r t C|^{2}\left|e^{i \phi_{1}}+e^{i \phi_{2}}\right|^{2}=2|r t C|^{2}(1+\cos \theta), \theta=\phi_{1}-\phi_{2}$. This implies the variation of the measured coincidence rate with signal path delay shown in Figure 3, with the oscillation period determined by the signal path delay $\left(\phi_{1}\right)$ and therefore the 808-nm signal wavelength.

If $C_{1} \neq C_{2}$, which would be the case if, for example, the pump amplitudes at the two crystals were different, the coincidence rate $R_{A B}$ as discussed above will be proportional to $|r t|^{2}\left|C_{1} e^{i \phi_{1}}+C_{2} e^{i \phi_{2}}\right|^{2}$. Writing $C_{2}=\beta C_{1}=|\beta| e^{i \theta_{\beta}}$, we have

$$
R_{A B} \propto\left|r t C_{1}\right|^{2}\left[1+|\beta|^{2}+2|\beta| \cos \theta_{\beta}\right]
$$

and a coincidence-rate "visibility":

$$
V=\frac{R_{A B}^{\max }-R_{A B}^{\min }}{R_{A B}^{\max }+R_{A B}^{\min }}=\frac{2|\beta|}{1+|\beta|^{2}}
$$

We similarly define a contrast, or "distinguishability",

$$
K \equiv \frac{\left|C_{1}\right|^{2}-\left|C_{2}\right|^{2}}{\left|C_{1}\right|^{2}+\left|C_{2}\right|^{2}}=\frac{1-|\beta|^{2}}{1+|\beta|^{2}}
$$

It follows that

$$
K^{2}+V^{2}=1
$$

which is a special case of the more general expression $K^{2}+V^{2} \leq 1$ of complementarity [15-19]. The inequality in our case would apply if we did not make the implicit assumption that the pump fields at the two crystals are mutually coherent.

First-order perturbation theory with the interaction Hamiltonian (4), assuming an initial state $\left|0_{s 1} 0_{i 1}\right\rangle\left|0_{s 2} 0_{i 2}\right\rangle$ with no photons in the modes s1,i1,s2,i2 (Figure 1 ), gives

$$
|\psi\rangle=\left|0_{s 1} 0_{i} 1\right\rangle\left|0_{s 2} 0_{i 2}\right\rangle+K\left(C_{1}\left|1_{s 1} 1_{i 1}\right\rangle\left|0_{s 2} 0_{i 2}\right\rangle+C_{2}\left|0_{s 1} 0_{i 1}\right\rangle\left|1_{s 2} 1_{i 2}\right\rangle\right) .
$$

Here, $\left|1_{s 1} 1_{i 1}\right\rangle$ is the state with one photon in each of the modes $s 1$ and $i 1$, etc. The factor $K$ need not be specified, nor will it be necessary to normalize $|\psi(t)\rangle$ for our purposes. As discussed in [8], the "entanglement with the vacuum" described by the state (9) implies the observed two-photon coincidence rate. In terms of interfering probability amplitudes as discussed above, the amplitude to count (annihilate) a signal photon $s 1$ at Detector A in coincidence with an idler photon $i 1$ at Detector B, including the phase delay $\phi_{1}$, is proportional to $\left\langle\operatorname{vac}\left|a_{s 1} e^{i \phi_{1}} a_{i 1}\right| \psi\right\rangle=C_{1} e^{i \phi_{1}}$, where the vacuum state of the field is denoted by

$$
\mid \text { vac }\rangle=\left|0_{s 1} 0_{i 1}\right\rangle\left|0_{s 2} 0_{i 2}\right\rangle .
$$

Similarly, the amplitude to count a signal photon $s 2$ at Detector $A$ in coincidence with an idler photon $i 2$ at Detector B is proportional to $\left\langle\operatorname{vac}\left|a_{s 2} a_{i 2} e^{i \phi_{2}}\right| \psi\right\rangle=C_{2} e^{i \phi_{2}}$, and the rate for counting a signal 
photon at $\mathrm{A}$ in coincidence with an idler photon at $\mathrm{B}$ is therefore proportional to $\left|C_{1} e^{i \phi_{1}}+C_{2} e^{i \phi_{2}}\right|^{2}$. This simple argument emphasizes the role played by entanglement with the vacuum field: to explain the observed coincidence rate, we must include the vacuum states $\left|0_{s 1} 0_{i 1}\right\rangle$ and $\left|0_{s 2} 0_{i 2}\right\rangle$, as well as the two-photon states $\left|1_{s 1} 1_{i 1}\right\rangle$ and $\left|1_{s 2} 1_{i 2}\right\rangle$ in describing the state of the field.

We now turn to a simplified Heisenberg picture analysis that exhibits explicitly the role of the vacuum fields, as opposed to the vacuum states, participating in the downconversion at the two crystals. The rate for counting a signal photon at Detector A in coincidence with an idler photon at Detector B may be taken to be proportional to [20,21]

$$
R_{A B}=\left\langle\operatorname{vac}\left|E_{A}^{(-)} E_{B}^{(-)} E_{B}^{(+)} E_{A}^{(+)}\right| \mathrm{vac}\right\rangle,
$$

where $E_{A}^{(+)}$and $E_{B}^{(+)}$are the positive-frequency parts of the electric field operators at Detectors A and $\mathrm{B}, E_{A}^{(-)}, E_{B}^{(-)}$are their Hermitian conjugates, and $|\mathrm{vac}\rangle$ is again the vacuum field state. As in the preceding discussion, we treat $\mathrm{A}$ and $\mathrm{B}$ as effectively point detectors, since as a practical matter, the spatial integration over detector volumes only introduces a complication of no interest for our purposes here. We therefore ignore entirely the spatial variations of the electric fields, except, of course, for the path delays described by $\phi_{1}$ and $\phi_{2}$. Then, to calculate the dependence of $R_{A B}$ on these path delays, we can effectively take, to first-order in $D$,

$$
E_{A}^{(+)}=\left(\left[a_{s 10}+D a_{i 10}^{+}\right] r e^{i \phi_{1}}+\left[a_{s 20}+D a_{i 20}^{\dagger}\right] t\right) e^{-i \omega_{s} t}
$$

and

$$
E_{B}^{(+)}=\left(\left[a_{i 10}+D a_{s 10}^{\dagger}\right] t+\left[a_{i 20}+D a_{s 20}^{\dagger}\right] r e^{i \phi_{2}}\right) e^{-i \omega_{i} t},
$$

which are obvious extensions of (2) and (3).

Suppose first that only the crystal BBO1 is pumped. Since $a_{s 10}|\mathrm{vac}\rangle=a_{s 20}|\mathrm{vac}\rangle=a_{i 10}|\mathrm{vac}\rangle=$ $a_{i 20}|\mathrm{vac}\rangle=0$, and the photon operators for different modes commute,

$$
\left\langle E_{B}^{(-)} E_{A}^{(+)}\right\rangle=0,
$$

i.e., the signal and idler fields in SPDC are uncorrelated [7]. The signal-idler photon coincidence rate is found similarly; to second-order in D,

$$
R_{A B}=|D|^{2}|r t|^{2}\left\langle\operatorname{vac}\left|a_{i 10} a_{i 10}^{+} a_{i 10} a_{i 10}^{+}\right| \mathrm{vac}\right\rangle=|D|^{2}|r t|^{2} .
$$

The dependence of this signal-idler coincidence rate on only the vacuum idler field reflects the fact that a signal photon at Detector A, produced by the mixing of the vacuum idler field with the pump field at BBO1, is accompanied by its partner idler photon at Detector B. Since $\left[E_{A}^{(+)}, E_{B}^{(+)}\right]=0$, we can also write $R_{A B}$ in the form

$$
R_{A B}=\left\langle\operatorname{vac}\left|E_{B}^{(-)} E_{A}^{(-)} E_{A}^{(+)} E_{B}^{(+)}\right| \mathrm{vac}\right\rangle=|D|^{2}|r t|^{2}\left\langle\operatorname{vac}\left|a_{s 10} a_{s 10}^{+} a_{s 10} a_{s 10}^{+}\right| \mathrm{vac}\right\rangle=|D|^{2}|r t|^{2},
$$

which can be interpreted in the same way as (15), except that now, the roles of the signal and idler fields are switched. The fact that we can write $R_{A B}$ in terms of either signal or idler vacuum expectation values simply reflects the fact that signal and idler photons are generated in (biphoton) pairs.

For the experiment of Figure 1 in which both crystals are pumped, we obtain, from (11)-(13),

$$
\begin{aligned}
R_{A B}= & |D|^{2}|r t|^{2}\left(\left\langle\operatorname{vac}\left|a_{i 10} a_{i 10}^{\dagger} a_{i 10} a_{i 10}^{\dagger}\right| \mathrm{vac}\right\rangle+\left\langle\operatorname{vac}\left|a_{i 20} a_{i 20}^{\dagger} a_{i 20} a_{i 20}^{\dagger}\right| \mathrm{vac}\right\rangle\right. \\
& \left.+2\left\langle\operatorname{vac}\left|a_{i 10} a_{i 10}^{\dagger} a_{i 20} a_{i 20}^{\dagger}\right| \mathrm{vac}\right\rangle \cos \theta\right)
\end{aligned}
$$


to lowest order in $D$, where again, $\theta=\phi_{1}-\phi_{2}$. Equivalently, since $\left[E_{A}^{(+)}, E_{B}^{(+)}\right]=0$,

$$
\begin{aligned}
R_{A B}= & |D|^{2}|r t|^{2}\left(\left\langle\operatorname{vac}\left|a_{s 10} a_{s 10}^{\dagger} a_{s 10} a_{s 10}^{\dagger}\right| \mathrm{vac}\right\rangle+\left\langle\operatorname{vac}\left|a_{s 20} a_{s 20}^{\dagger} a_{s 20} a_{s 20}^{+}\right| \mathrm{vac}\right\rangle\right. \\
& \left.+2\left\langle\operatorname{vac}\left|a_{s 10} a_{s 10}^{\dagger} a_{s 20} a_{s 20}^{\dagger}\right| \mathrm{vac}\right\rangle \cos \theta\right)
\end{aligned}
$$

Since all the vacuum expectation values in (16) and (17) $=1$, we obtain again $R_{A B} \propto 1+\cos \theta$ :

$$
R_{A B}=2|D|^{2}|r t|^{2}(1+\cos \theta)
$$

The generalization to non-identical crystals or pump fields is straightforward and of course reproduces the complementarity relation (8).

In the derivation of $R_{A B}$ based on probability amplitudes, the $\cos \theta$ term resulted from the interference of the amplitudes for the two indistinguishable processes (i) and (ii). Obviously, the interference here is similar to the interference of two probability amplitudes in the two-slit experiment with single photons, with the difference that $R_{A B}$ describes the counting of two photons (signal and idler) in coincidence. The complementarity relation (8) applies to both examples. In the classical description of the two-slit experiment, for example, the visibility of interference fringes is reduced when the difference in the field intensities incident on the two slits is increased. In the experiment of Figure 1, the visibility (6) is reduced when the distinguishability of the processes (i) and (ii) is increased.

In the Heisenberg picture calculation, the role of the vacuum fields taking part in SPDC is explicit in Expression (16). The vacuum fields incident on BBO1 and BBO2 are associated with $a_{i 10}$ and $a_{i 20}$, respectively, and the last term on the right-hand side of (16) is non-vanishing because these fields have quantum fluctuations and zero-point energy, e.g., $\left\langle\mathrm{vac}\left|\frac{1}{2} \hbar \omega_{i} a_{i 10} a_{i 10}^{\dagger}\right| \mathrm{vac}\right\rangle=\frac{1}{2} \hbar \omega_{i}$. This suggests an interpretation along the lines of the classical theory of stochastic electrodynamics, which we discuss in Section 7.

\section{Vacuum Fields and the Hong-Ou-Mandel Dip}

The same simplified formalism in which the vacuum fields play an explicit role can be used to describe the Hong-Ou-Mandel-dip [13] (Figure 6). In this case, photons can be counted in coincidence at Detectors A and B if (i) both signal and idler fields are reflected by the symmetric beam splitter (BS), or (ii) both signal and idler fields are transmitted by the beam splitter. The probability amplitudes for these indistinguishable processes are proportional to $r^{2}$ and $t^{2}$, respectively. The coincidence rate is proportional to $\left|r^{2}+t^{2}\right|^{2}$, and a dip in the coincidence rate $\left(R_{A B}=0\right)$ is then obtained when $|r|=|t|=1 / \sqrt{2}$, since $r=t e^{i \pi / 2}$.

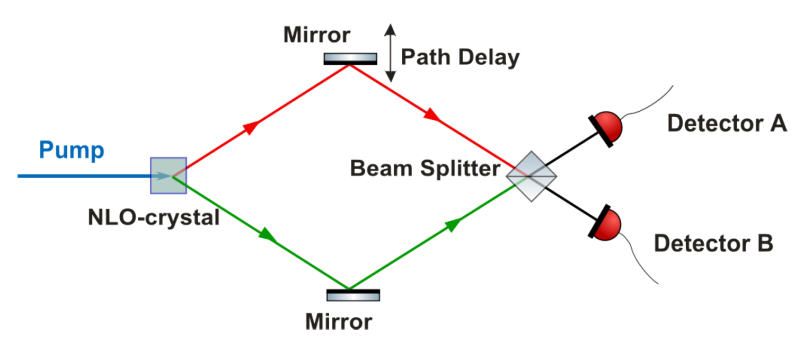

Figure 6. Hong-Ou-Mandel setup for observation of a dip in the signal-idler photon coincidence rate at Detectors A and B. The signal and idler fields, which are assumed here to have the same frequency and polarization, are reflected by the upper and lower mirrors, respectively and can either be reflected or transmitted by the beam splitter (BS). 
We can also relate the HOM interference effect to "entanglement with the vacuum" by writing the field state given by perturbation theory as

$$
|\psi\rangle=\left|0_{s} 0_{i}\right\rangle_{A}\left|0_{S} 0_{i}\right\rangle_{B}+D t^{2}\left|1_{s} 1_{i}\right\rangle_{A}\left|0_{s} 0_{i}\right\rangle_{B}+D r^{2}\left|0_{s} 0_{i}\right\rangle_{A}\left|1_{s} 1_{i}\right\rangle_{B}
$$

similarly to (9), where now, A and B refer to field modes incident at A and B. The transition amplitudes implied by this state imply $R_{A B} \propto\left|r^{2}+t^{2}\right|^{2}$, as above.

In our simplified Heisenberg picture approach, the positive-frequency parts of the electric field operators at Detectors A and B are

$$
E_{A}^{(+)}=\left(r\left(a_{s 0}+D a_{i 0}^{\dagger}\right) e^{-i \omega_{s} t}+t\left(a_{i 0}+D a_{s 0}^{\dagger}\right) e^{-i \omega_{i} t}\right)
$$

and

$$
E_{B}^{(+)}=\left(t\left(a_{s 0}+D a_{i 0}^{\dagger}\right) e^{-i \omega_{s} t}+r\left(a_{i 0}+D a_{s 0}^{\dagger}\right) e^{-i \omega_{i} t}\right),
$$

where the signal and idler fields are assumed to traverse the same distance. Evaluating $R_{A B}=$ $\left\langle\operatorname{vac}\left|E_{A}^{(-)} E_{B}^{(-)} E_{B}^{(+)} E_{A}^{(+)}\right| \mathrm{vac}\right\rangle$ in the same way as for the two-crystal experiment, we obtain, to lowest order in $D$,

$$
\begin{aligned}
R_{A B}= & \left(|t|^{4}\left\langle\operatorname{vac}\left|a_{s 0} a_{s 0}^{\dagger} a_{s 0} a_{s 0}^{\dagger}\right| \mathrm{vac}\right\rangle+|r|^{4}\left\langle\operatorname{vac}\left|a_{i 0} a_{i 0}^{\dagger} a_{i 0} a_{i 0}^{\dagger}\right| \mathrm{vac}\right\rangle\right. \\
& \left.+r^{2} t^{* 2}\left\langle\operatorname{vac}\left|a_{s 0} a_{s 0}^{\dagger} a_{i 0} a_{i 0}^{\dagger}\right| \mathrm{vac}\right\rangle+r^{* 2} t^{2}\left\langle\operatorname{vac}\left|a_{i 0} a_{i 0}^{\dagger} a_{s 0} a_{s 0}^{\dagger}\right| \mathrm{vac}\right\rangle\right)|D|^{2} \\
= & \left|r^{2}+t^{2}\right|^{2}|D|^{2},
\end{aligned}
$$

since the vacuum expectation values are all one. Since $\left[E_{A}^{(+)}, E_{B}^{(+)}\right]=0$, we can also write

$$
\begin{aligned}
R_{A B}= & \left(|t|^{4}\left\langle\operatorname{vac}\left|a_{i 0} a_{i 0}^{\dagger} a_{i 0} a_{i 0}^{\dagger}\right| \mathrm{vac}\right\rangle+|r|^{4}\left\langle\operatorname{vac}\left|a_{s 0} a_{s 0}^{\dagger} a_{s 0} a_{s 0}^{\dagger}\right| \text { vac }\right\rangle\right. \\
& \left.+r^{2} t^{* 2}\left\langle\operatorname{vac}\left|a_{s 0} a_{s 0}^{\dagger} a_{i 0} a_{i 0}^{\dagger}\right| \text { vac }\right\rangle+r^{* 2} t^{2}\left\langle\operatorname{vac}\left|a_{i 0} a_{i 0}^{\dagger} a_{s 0} a_{s 0}^{\dagger}\right| \text { vac }\right\rangle\right)|D|^{2} .
\end{aligned}
$$

In either form, the HOM dip $\left(R_{A B}=0\right)$ then follows for $|r|=|t|=1 / \sqrt{2}$ and the fact that $r=t e^{i \pi / 2}$ for the symmetric beam splitter.

These expressions have some similarity to those encountered in the theory of the Hanbury Brown-Twiss intensity interference of chaotic classical fields. Thus, the first and second terms in (23), for instance, correspond in effect to mean-squared intensities, while the third and fourth terms effectively correspond to a (second-order) signal-idler "intensity" interference and are nonvanishing even though there is no (first-order) interference of the signal and idler fields. The difference in the HOM case is that the interference may be thought of as occurring between the signal and idler vacuum field "intensities" proportional to $\left\langle a_{s 0} a_{s 0}^{\dagger}\right\rangle$ and $\left\langle a_{i 0} a_{i 0}^{\dagger}\right\rangle$, respectively.

\section{Remarks on the Simplified Theory}

The theoretical approach we have taken accounts for the experimental results described in Section 3, as well as the HOM dip, and as discussed in Section 4, it is consistent with the basic notions of complementarity, which-path information, and the interference of probability amplitudes. It also accounts for the main results of earlier experiments involving three crystals, induced coherence, and stimulated down-conversion $[7,10,12]$. In addition to the assumption of monochromatic pump and signal and idler fields, the applicability of this simplified theory relies on the assumption that the 
SPDC rate of generation of biphotons is very small. Consider, for example, the approximate solution for the signal photon annihilation operator $a_{s}$ implied by (2):

$$
a_{s} \cong\left(a_{s 0}+D a_{i 0}^{\dagger}\right) e^{-i \omega_{s} t} .
$$

Since vacuum-field signal and idler field operators satisfy $\left[a_{s 0}, a_{s 0}^{\dagger}\right]=\left[a_{i 0}, a_{i 0}^{\dagger}\right]=1,\left[a_{s 0}, a_{i 0}\right]=$ $\left[a_{s 0}, a_{i 0}^{\dagger}\right]=0,(24)$ implies the incorrect equal-time commutation relation

$$
\left[a_{s}, a_{s}^{\dagger}\right] \cong 1-|D|^{2}
$$

In a more complete theory in which the down-conversion efficiency is not necessarily small, $a_{s}$ is related to $a_{s 0}$ and $a_{i 0}^{\dagger}$ by a Bogoliubov transformation [22]:

$$
a_{s}=u_{s} a_{s 0}+v_{s} a_{i 0}^{\dagger}, \quad\left|u_{s}\right|^{2}-\left|v_{s}\right|^{2}=1,
$$

and likewise for $a_{i}$, and the correct commutation relations $\left[a_{s}, a_{s}^{\dagger}\right]=\left[a_{i}, a_{i}^{\dagger}\right]=1$ are satisfied. To better appreciate the approximation (24) (and the corresponding approximation for $a_{i}$ ), consider first the expectation value of the number of signal photons implied by (26):

$$
\left\langle n_{s}\right\rangle=\left\langle\operatorname{vac}\left|a_{s}^{\dagger} a_{s}\right| \operatorname{vac}\right\rangle=\left|v_{s}\right|^{2}\left\langle\operatorname{vac}\left|a_{i 0} a_{i 0}^{\dagger}\right| \mathrm{vac}\right\rangle=\left|v_{s}\right|^{2} .
$$

Next, consider the expectation value of the square of the number of signal photons implied by (26):

$$
\begin{aligned}
\left\langle n_{s}^{2}\right\rangle & =\left\langle\operatorname{vac}\left|a_{s}^{\dagger} a_{s} a_{s}^{\dagger} a_{s}\right| \mathrm{vac}\right\rangle \\
& =\left|u_{s}\right|^{2}\left|v_{s}\right|^{2}\left\langle\mathrm{vac}\left|a_{s 0} a_{s 0}^{\dagger} a_{i 0} a_{i 0}^{\dagger}\right| \mathrm{vac}\right\rangle+\left|v_{s}\right|^{4}\left\langle\operatorname{vac}\left|a_{i 0} a_{i 0}^{\dagger} a_{i 0} a_{i 0}^{\dagger}\right| \mathrm{vac}\right\rangle \\
& =\left|u_{s}\right|^{2}\left|v_{s}\right|^{2}+\left|v_{s}\right|^{4} .
\end{aligned}
$$

Now, if, as in the experiments described earlier, the probability of generating more than one biphoton in the measurement interval is very small, we can assume that $\left\langle n_{s}^{2}\right\rangle=\left\langle n_{s}\right\rangle=0$ or 1, i.e., that there is at most a single signal-idler pair generated during the measurement interval. This means that (28) should be well approximated by (27), which in turn implies that $\left|u_{s}\right|^{2} \cong 1$ and $\left|v_{S}\right|^{2} \ll 1$. Comparison of (24) and (26) then implies that $|D| \ll 1$. The simplified theory is therefore accurate as long as, with high probability, there is at most a single biphoton during the measurement interval, and we therefore require that $|D| \ll 1$.

The relation (25) in our simplified theory does not invalidate our calculations because we have not required $\left[a_{s}^{\dagger}, a_{s}\right]=\left[a_{i}^{\dagger}, a_{i}\right]=1$ anywhere in these calculations. We have, however, used the commutation relation $\left[E_{A}^{(+)}, E_{B}^{(+)}\right]=0$ in writing (17), for instance, but this is perfectly consistent within the simplified theory, since, from (12) and (13),

$$
\left[E_{A}^{(+)}, E_{B}^{(+)}\right]=D r^{2}\left(\left[a_{s 0}, a_{s 0}^{\dagger}\right]+\left[a_{i 0}^{\dagger}, a_{i 0}\right]+D t^{2}\left[a_{i 0}, a_{i 0}^{\dagger}\right]+\left[a_{s 0}^{\dagger}, a_{s 0}\right]\right)=0,
$$

for all values of $r$ and $t$.

\section{Classical Stochastic Theory of Vacuum Fields}

Signal-idler intensity correlations in single-crystal SPDC have been shown to follow from a theory in which the incoming vacuum signal fields are treated as classical stochastic fields [11]. We now re-examine such an approach, following the same lines as the simplified theory we have used in treating these correlations with quantized fields. Our approach will be semiclassical in the sense that the fields are treated classically, while the photodetection is described by replacing normally-ordered field correlation functions such as $\left\langle E^{(-)}\left(\mathbf{r}_{1}, t_{1} E^{(+)}\left(\mathbf{r}_{2}, t_{2}\right)\right\rangle\right.$ in the quantized-field theory by $\left\langle\left\langle\mathcal{E}^{(-)}\left(\mathbf{r}_{1}, t_{1}\right) \mathcal{E}^{(+)}\left(\mathbf{r}_{2}, t_{2}\right)\right\rangle\right\rangle$, where $\langle\langle\ldots\rangle\rangle$ denotes an average over an appropriate classical 
ensemble. This approach is justified for our purposes by the fact that the main features of photoelectric detection do not require field quantization [23]. The same approach is in fact implicit in the part of the work of [11] that relates to the classical stochastic description of the vacuum fields.

For a single vacuum mode of frequency $\omega$, the positive-frequency part of the classical stochastic field is assumed, as in the completely classical theory of stochastic electrodynamics [24,25], to have the form

$$
\mathcal{E}^{(+)}(\mathbf{r}, t)=\alpha e^{i \theta} e^{-i \omega t},
$$

such that $\alpha$ is a fixed constant and $\theta$ is a random variable having a uniform probability distribution on the interval $[0,2 \pi]$. The appropriate averaging $\langle\langle\ldots\rangle\rangle$ is then an average over the phase distribution, and it is assumed that the randomly-varying phases of different modes are uncorrelated. We can fix the values of the $\alpha$ 's such that the average energy density per mode in the classical stochastic theory is the same as the corresponding quantized-field vacuum expectation value, which for our purposes is proportional to

$$
\left\langle\operatorname{vac}\left|\left[E^{(+)}+E^{(-)}\right]^{2}\right| \operatorname{vac}\right\rangle=\left\langle\operatorname{vac}\left|\left[a e^{-i \omega t}+a^{\dagger} e^{i \omega t}\right]^{2}\right| \operatorname{vac}\right\rangle=\left\langle\operatorname{vac}\left|a a^{\dagger}\right| \operatorname{vac}\right\rangle=1 .
$$

Since

$$
\left\langle\left\langle\left[\mathcal{E}^{(+)}+\mathcal{E}^{(-)}\right]^{2}\right\rangle\right\rangle=\left\langle\left\langle\left[\alpha e^{i \theta} e^{-i \omega t}+\alpha^{*} e^{-i \theta} e^{i \omega t}\right]^{2}\right\rangle\right\rangle=2|\alpha|^{2},
$$

this requires

$$
|\alpha|^{2}=1 / 2
$$

for each field mode in the stochastic theory. As in the preceding calculations, it will suffice for our purposes to ignore any explicit consideration of the spatial dependence of the fields.

The classical counterparts of the quantized fields (12) and (13) are

$$
\mathcal{E}_{A}^{(+)}=\left(\left[\alpha_{s 10} e^{i \theta_{s 10}}+D \alpha_{i 10}^{*} e^{-i \theta_{i 10}}\right] r e^{i \phi_{1}}+\left[\alpha_{s 20} e^{i \theta_{s 20}}+D \alpha_{i 20}^{*} e^{-i \theta_{i 20}}\right] t\right) e^{-i \omega_{s} t}
$$

and

$$
\mathcal{E}_{B}^{(+)}=\left(\left[\alpha_{i 10} e^{i \theta_{i 10}}+D \alpha_{s 10}^{*} e^{-i \theta_{s 10}}\right] t+\left[\alpha_{i 20} e^{i \theta_{i 20}}+D \alpha_{s 20}^{*} e^{-i \theta_{s 20}}\right] r e^{i \phi_{2}}\right) e^{-i \omega_{i} t},
$$

where $\theta_{s 10}, \theta_{i 10}, \theta_{s 20}$, and $\theta_{i 20}$ are uncorrelated random variables, uniformly distributed on $[0,2 \pi]$.

Suppose first that only the crystal BBO1 in Figure 1 is pumped. The fields at A and B are uncorrelated because of their random and uncorrelated phases, and this is consistent with the quantized-field theory. For the signal-idler coincidence rate, we obtain

$$
\begin{aligned}
\mathcal{R}_{A B}= & \left\langle\left\langle\mathcal{E}_{A}^{(-)} \mathcal{E}_{A}^{(+)} \mathcal{E}_{B}^{(-)} \mathcal{E}_{B}^{(+)}\right\rangle\right\rangle \\
= & \left|\alpha_{i 10}\right|^{2}\left|\alpha_{s 10}\right|^{2}|r|^{2}|t|^{2} \\
& +|D|^{2}|r|^{2}|t|^{2}\left(2\left|\alpha_{i 10}\right|^{2}\left|\alpha_{s 10}\right|^{2}+\left|\alpha_{s 10}\right|^{4}+\left|\alpha_{i 10}\right|^{4}\right)
\end{aligned}
$$

in the stochastic theory. If we ignore the first, $D$-independent term and replace each $|\alpha|^{2}$ in the remaining term by $1 / 2$ as in (33), we obtain

$$
\mathcal{R}_{A B}=|D|^{2}|r|^{2}|t|^{2},
$$


in agreement with the result (16) obtained in the quantized-field theory. In the case that both crystals in Figure 1 are pumped, we obtain, similarly,

$$
\begin{aligned}
\mathcal{R}_{A B}= & \left(\left|\alpha_{s 10}\right|^{2}\left|\alpha_{i 20}\right|^{2}|r|^{4}+\left|\alpha_{s 20}\right|^{2}\left|\alpha_{i 10}\right|^{2}|t|^{4}+\left|\alpha_{s 20}\right|^{2}\left|\alpha_{i 20}\right|^{2}|r|^{2}|t|^{2}+\left|\alpha_{s 10}\right|^{2}\left|\alpha_{i 10}\right|^{2}|r|^{2}|t|^{2}\right) \\
& +\left(\left|\alpha_{i 10}\right|^{4}+\left|\alpha_{i 20}\right|^{4}+\left|\alpha_{s 10}\right|^{4}+\left|\alpha_{s 20}\right|^{4}+2\left|\alpha_{i 10}\right|^{2}\left|\alpha_{s 10}\right|^{2}+2\left|\alpha_{i 20}\right|^{2}\left|\alpha_{s 20}\right|^{2}\right)|D|^{2}|r|^{2}|t|^{2} \\
& +2\left(\left|\alpha_{i 10}\right|^{2}\left|\alpha_{i 20}\right|^{2}+\left|\alpha_{s 10}\right|^{2}\left|\alpha_{i 20}\right|^{2}+\left|\alpha_{s 10}\right|^{2}\left|\alpha_{i 20}\right|^{2}+\left|\alpha_{s 20}\right|^{2}\left|\alpha_{i 10}\right|^{2}\right)|D|^{2}|r|^{2}|t|^{2} \cos \theta
\end{aligned}
$$

where again $\theta=\phi_{1}-\phi_{2}$. If we again ignore the $D$-independent term and replace every $|\alpha|^{2}$ by $1 / 2$, we obtain

$$
\mathcal{R}_{A B}=2(1+\cos \theta)|D|^{2}|r|^{2}|t|^{2},
$$

in agreement with the result (18) derived in the quantized-field theory.

$D$, which was introduced in Section 4, is proportional to the lowest (second-) order nonlinear susceptibility, the pump-field amplitude, and the interaction time of the fields in the crystals, and it has been assumed for simplicity that it is the same for each crystal. In most SPDC experiments, $|D| \ll 1$, as we have assumed, so that the generation of multiple biphotons during the interaction time is negligible. (The assumption that the generation of multiple biphotons during the interaction time is negligible is also made in the more detailed, multimode analysis of Hong and Mandel [26].) As shown in Section $6, D \ll 1$ is in fact required for the validity of our simplified theory.

The $D$-independent terms we have ignored in obtaining (37) and (39) arise from the signal and idler vacuum fields that are present even in the absence of any pump field or crystals. In free space without any crystals, for example, (30) implies that $\left\langle\left\langle\mathcal{E}^{(-)} \mathcal{E}^{(+)}\right\rangle\right\rangle=|\alpha|^{2}$, i.e., a photodetector would respond to a vacuum field in free space without any sources. It would make sense, therefore, to argue that the physically meaningful, measurable intensity should be defined by subtracting out the source-free contribution in the classical stochastic treatment of the vacuum field [11,27]. In the calculation of $\mathcal{R}_{A B}$, it might be argued similarly that the $D$-independent terms in (34) and (35) are unphysical and should be dropped. A problem with such an argument, however, is that those terms contribute to the $D$-dependent terms in $R_{A B}$, and without them, the stochastic theory does not reproduce results such as (37) or (39) that are consistent with the quantized-field theory and with the experiment, as discussed earlier. In other words, the $D$-independent terms are required if we are to obtain interference effects that appear naturally in the quantized-field theory. Thus, while a classical stochastic approach to the vacuum field can yield results that mimic those found in the quantized-field theory, such an approach appears to be fundamentally inconsistent.

In the quantized-field theory, there are also, of course, $D$-independent operators in the expressions for $E_{A}^{( \pm)}$and $E_{B}^{( \pm)}$, and they do contribute explicitly to $R_{A B}$. However, in the quantized-field theory, these operators do not give rise to measurable effects in the absence of any sources, nor do they give rise to $D$-independent terms in $\mathcal{R}_{A B}$. Unlike the intensity $\left\langle\left\langle\mathcal{E}^{(-)} \mathcal{E}^{(+)}\right\rangle\right\rangle=|\alpha|^{2}$ in the absence of any sources, for example, $\left\langle E^{(-)} E^{(+)}\right\rangle=\mid\left\langle\mathrm{vac}\left|a^{\dagger} a\right|\right.$ vac $\rangle=0$ in the quantized-field theory: a photodetector does not respond to a source-free, vacuum field.

\section{Concluding Remarks}

The high-visibility interference of the biphotons from two parallel pumped, but otherwise completely decoupled crystals shown in Figure 4 can be intuitively understood with a model based on signal and idler vacuum fields. The vacuum signal and idler fields not only mix with the pump field to generate the biphotons; the "un-mixed" parts of these fields in the same biphoton modes also experience phase delays as they propagate to the detectors and act with the generated fields to give the measured coincidence counting rate. This model leads to the same predictions as the conventional picture emphasizing entanglement, complementarity, and which-path information in the coincidence counting of signal photons at Detector A and idler photons at Detector B. 
We have also shown with our simplified model that the Hong-Ou-Mandel dip can be described not only as an interference effect resulting from entanglement with the vacuum state, but also as a consequence of vacuum fields. As in the simplified theory of the two-crystal experiment, the expressions we obtained for the HOM interference (Equations (22) and (23)) involve only vacuum fields.

The vacuum-field interpretations discussed in this paper are not the only ones possible. Our intention here has only been to show that, within the context of spontaneous parametric down-conversion, the vacuum-field picture allows for a simple, alternative, and fairly intuitive approach to the understanding of results obtained in different experimental configurations. While this approach suggests an interpretation along the lines of a classical stochastic theory of the vacuum field, we have argued that such a theory is fundamentally inconsistent because it predicts that there would be photon coincidence counting over and above what is observed and that such coincidence counts would be observed even in the absence of the pump fields.

Author Contributions: All three authors contributed to the conceptualization of this work. A.H. and R.M. developed the methodology and the experiments were done by A.H. The formal theory was developed by P.W.M. and all three authors contributed to the analysis, writing and editing.

Funding: This research received no external funding.

Conflicts of Interest: The authors declare no conflicts of interest.

\section{References}

1. Gisin, N.; Ribordy, G.; Tittel, W.; Zbinden, H. Quantum cryptography. Rev. Mod. Phys. 2002, 74, 145. [CrossRef]

2. Malik, M.; Boyd, R.W. Quantum imaging technologies. Rivista del Nuovo Cimento 2014, 37, $273-332$.

3. Dorfman, K.E.; Schlawin, F.; Mukamel, S. Nonlinear optical signals and spectroscopy with quantum light. Rev. Mod. Phys. 2016, 88, 045008. [CrossRef]

4. Cho, M., Department of Chemistry, Korea University, Private Communication.

5. Giovannetti, V.; Lloyd, S.; Maccone, L. Advances in quantum metrology. Nat. Photonics 2011, 5, $222-229$. [CrossRef]

6. Mandel, L. Quantum effects in one-photon and two-photon interference. Rev. Mod. Phys. 1999, 71, S274. [CrossRef]

7. Heuer, A.; Menzel, R.; Milonni, P.W. Complementarity in biphoton generation with stimulated or induced coherence. Phys. Rev. A 2015, 92, 033834. [CrossRef]

8. Ou, Z.Y.; Wang, L.J.; Mandel, L. Vacuum effects in 2-photon downconversion. Phys. Rev. A 1989, 40, 1428. [CrossRef]

9. Ou, Z.Y.; Wang, L.J.; Zou, X.Y.; Mandel, L. Evidence for phase memory in two-photon down conversion through entanglement with the vacuum. Phys. Rev. A 1990, 41, 566. [CrossRef]

10. Heuer, A.; Menzel, R.; Milonni, P.W. Induced Coherence, Vacuum Fields, and Complementarity in Biphoton Generation. Phys. Rev. Lett. 2015, 114, 053601. [CrossRef]

11. Dechoum, K.; Marshall, T.W.; Santos, E. Parametric up and down conversion in the Wigner representation of quantum optics. J. Mod. Opt. 2000, 47, 1273-1287. [CrossRef]

12. Heuer, A.; Raabe, S.; Menzel, R. Phase memory across two single-photon interferometers including wavelength conversion. Phys. Rev. A 2014, 90, 045803. [CrossRef]

13. Hong, C.K.; Ou, Z.Y.; Mandel, L. Measurement of subpicosecond time intervals between 2 photons by interference. Phys. Rev. Lett. 1987, 59, 2044. [CrossRef] [PubMed]

14. Horne, M.A.; Shimony, A.; Zeilinger, A. 2-particle interferometry. Phys. Rev. Lett. 1989, 62, 2209. [CrossRef] [PubMed]

15. Glauber, R.J. Amplifiers, Attenuators, and Schrödinger's Cat. In New Techniques and Ideas in Quantum Measurement Theory; Greenberger, D.M., Ed.; New York Academy of Sciences: NY, USA, 1986; Volume 480, pp. 336-372.

16. Greenberger, D.M.; Yasin, A. Simultaneous wave and particle knowledge in a neutron interferometer. Phys. Lett. A 1988, 128, 391. [CrossRef]

17. Mandel, L. Coherence and indistinguishability. Opt. Lett. 1991, 16, 1882-1883. [CrossRef] 
18. Jaeger, G.; Shimony, A.; Vaidman, L. 2 interferometric complementarities. Phys. Rev. A 1995, 51, 54. [CrossRef] [PubMed]

19. Englert, B.G. Fringe visibility and which-way information: An inequality. Phys. Rev. Lett. 1996, 77, 2154. [CrossRef]

20. Glauber, R.J. Quantum theory of optical coherence. Phys. Rev. 1963, 130, 2529. [CrossRef]

21. Glauber, R. J. Coherent and incoherent states of the radiation field. Phys. Rev. 1963, 131, 2766. [CrossRef]

22. Braunstein, S.L. Squeezing as an irreducible resource. Phys. Rev. A 2005, 71, 055801. [CrossRef]

23. Scully, M.O.; Sargent, M. The concept of the photon. Phys. Today 1972, 25, 38. [CrossRef]

24. Boyer, T.H. A Brief Survey of Stochastic Electrodynamics. In Foundations of Radiation Theory and Quantum Electrodynamics; Barut, A.O., Ed.; Springer: New York, NY, USA, 1980; pp. 49-64.

25. de la Peña, L.; Cetto, A.M. The Quantum Dice: An Introduction to Stochastic Electrodynamics; Kluwer: Dordrecht, The Netherlands, 1996.

26. Hong, C.K.; Mandel, L. Theory of parametric frequency down conversion of light. Phys. Rev. A 1985, 31, 2409. [CrossRef]

27. Casado, A.; Marshall, T.W.; Santos, E. Parametric downconversion experiments in the Wigner representation. J. Opt. Soc. Am. B 1997, 14, 494-502. [CrossRef]

(C) 2019 by the authors. Licensee MDPI, Basel, Switzerland. This article is an open access article distributed under the terms and conditions of the Creative Commons Attribution (CC BY) license (http:/ / creativecommons.org/licenses/by/4.0/). 